\title{
Fuzzy-based Sentiment Analysis system for Analyzing Student Feedback and Satisfaction
}

Muhammad Zubair Asghar ${ }^{1}$, Ikram Ullah ${ }^{1}$, Shahaboddin Shamshirband ${ }^{2,3^{*}}$, Fazal Masud Kundi ${ }^{1}$, Ammara Habib ${ }^{1}$

${ }^{1}$ Institute of Computing and Information Technology, Gomal University, D.I.Khan. KP, Pakistan

2Department for Management of Science and Technology Development, Ton Duc Thang University, Ho Chi Minh City, Vietnam

${ }^{3}$ Faculty of Information Technology, Ton Duc Thang University, Ho Chi Minh City, Vietnam

Email of $1^{\text {st }}$ author author: zubair@gu.edu.pk

Email of $2^{\text {nd }}$ coolikram516@yahoo.com

Email of $3^{\text {rd }}$ author: shahaboddin.shamshirband@tdtu.edu.vn

Email of $4^{\text {th }}$ author: fmkundi@gmail.com

Email of $5^{\text {th }}$ author: ammara@gu.edu.pk

\begin{abstract}
The feedback collection and analysis has remained an important subject matter since long. The traditional techniques for student feedback analysis are based on questionnaire-based data collection and analysis. However, the student expresses their feedback opinions on online social media sites, which need to be analyzed. This study aims at the development of fuzzy-based sentiment analysis system for analyzing student feedback and satisfaction by assigning proper sentiment score to opinion words and polarity shifters present in the input reviews. Our technique computes the sentiment score of student feedback reviews and then applies fuzzy-logic module to analyze and quantify student's satisfaction at the fine-grained level. The experimental results reveal that the proposed work has outperformed the baseline studies as well as state-of-the-art machine learning classifiers.
\end{abstract}

Keywords: student feedback analysis; sentiments opinion words; polarity shifters; lexicon-based

\section{Introduction}

Sentiment Analysis (SA) also called opinion mining, is the field of study that analyzes people opinions, sentiments, evaluations, appraisals, attitude, and emotions towards entities such as 
products, services, organizations, individuals, issues, events, and topics. Due to the emergence of social media sites like Facebook, Twitter, and other web forums, online users express their sentiments which provide an important clue about their activities and feedback.

Like other online users, students also express their opinions on different sites, which provides an important clue in the form of feedback regarding their subjects, tutors and other facilities provided to them. Detection and classification of such feedback is important to be analyzed, as students express sentiments towards course, teaching faculty and other academic facilities, provided to them. It is also beneficial in terms of classifying student's feedback and quantifying their satisfaction, which can assist in improving the academic facilities provided to the students and preparation of annual confidential reports $[1,2]$.

The traditional techniques of classifying student feedback are not scalable, motivating researchers to develop automated techniques. In this work, we focus on the problem of classifying a student's review as satisfied, moderate, or not-satisfied. The task faces different challenges, such as different kinds of extremism, various targets and multiple ways of representing the same semantics. The existing studies of student feedback analysis are based on lexicon-based techniques [3] or use classical feature representation schemes followed by a classical machine learning classifier. However, recently fuzzy-based sentiment analysis techniques have yielded better results for complex problems in different domains such as business, healthcare, and others.

Fuzzy-based sentiment analysis is one of the feasible solutions for analyzing user feedback and satisfaction. In this technique scores of user sentiments are computed using different dictionaries of sentiment words annotated with their semantic orientation i.e. polarity and strength [1], and in next phase, fuzzy logic module can be applied to compute user satisfaction at different levels of granularities [4]. In this work, we present a novel technique of utilizing aggregated sentiment score of each student's feedback (review) and make it input to the fuzzy logic module for quantifying student feedback and satisfaction. For analyzing student feedback and satisfaction, we take the task of a fuzzy based sentiment analysis as a multi-label classification task. We define the reviews $R=\{r 1, r 2, r 3, \ldots . r n\}$, and a class tag (positive, negative and neutral) is assigned by using SentiWordNet lexicon. The aim is to design a fuzzy-based sentiment analysis model, which takes 
sentiment score as input and can measure the student satisfaction level as either satisfied, moderate, or not-satisfied. The response given by the students concerning their perceptions of the teacher, department, faculty etc. and other issues about their institution, can be used for the improvement of the education, and teaching staff. We propose a technique to identify and classify such content.

In this work, we propose to compute sentiment scores of opinion words and modifiers, and then use the aggregated sentiment score as an input to the fuzzy-logic module for analyzing and measuring the student feedback and satisfaction. As baselines, we compare with student feedback evaluation systems proposed by $[2,3,5]$, based on the lexicon entries and supervised learning, which lacks in providing evaluation of student feedback at fine-grained level. In this work, we answer following research questions: RQ1. How can we perform efficient classification of opinion words expressed by student in their feedback by revising the sentiment scoring technique proposed in baseline method?, RQ2. What is the efficiency of fuzzy-based student feedback sentiment classification system with and without considering polarity shifters? RQ3. What is the efficiency of fuzzy-based sentiment analysis of student feedback w.r.t to state-of-the-art work and different supervised Machine Learning Algorithms?

The contributions of this work are summarized as follows:

(i) Development of sentiment scoring technique for assigning proper polarity score to opinion words expressed by the students in their feedback.

(ii) Classification of polarity shifters with respect to opinion words in the student textual responses.

(iii) Development of fuzzy-based sentiment analysis system for analyzing student feedback and satisfaction.

(iv) Performance evaluation of the proposed system with respect to baseline method.

The rest of the article is organized as follows: section 2 present a review of literature; proposed methodology is presented in section 3; result and discussions are summarized in section 4; and the final chapter presents the conclusion and future work. 


\section{Related Work}

In this section, we present an overview of the selected studies on student feed-back analysis systems.

A sentiment-based teacher evaluation system is developed by [2], applying different text analysis techniques supported by lexicon. Their model overcomes the limitation traditional questionnairebased student evaluation techniques by providing an insight to the teacher performance using automated method. The sentiment at word-level are computed using word frequency and word polarity, to accumulate the sentiment score of student's over all attitude. The system used Nymi software and improved results are obtained with respect to base line method.

The machine learning and lexicon-based approach for collecting and analyzing student feedback with respect to performance evaluation of teachers si proposed by [3]. The proposed model was trained using lexicon-based and TF-IDF features for analyzing student sentiments expressed for teacher's performance evaluation. A dataset of 1230 student was acquired and an accuracy of $93 \%$ was achieved.

Yousif et al. [5] proposed a fuzzy-based computational model for measuring and classifying performance of teaching staff on the basis student feedback collected using questionnaires and surveys. Experimental results are encouraging showing performance improvement over the similar methods.

Pavani et al. [6] proposed fuzzy driven performance evaluation system, based on different factors. Fuzzy inference system is formulated to map given input to a given output fuzzy logic. Two membership functions are applied and compared and the function having little role in determining the +ive or-ive direction, is also reported.

Jyothi et al. [7] proposed a fuzzy-based performance evaluation model for analyzing performance of the teaching faculty in technical institutions. A set of rules and inference system is introduced. Fuzzification and defuzzifiction is applied to transform, analyze and interpret student feedback for performance evaluation. The model is helpful in preparing annual confidential reports 
The lexicon-based sentiment analysis system was proposed by [8] for automatic feedback analysis of student regarding teacher evaluation. Data is collected from 1148 student responses about 30 teachers, available publicly at www.ratemyprofessor.com. After applying different preprocessing steps, manually created lexicon is used to assign sentiment scores to opinion words. Different statistical techniques, such as Pearson's correlation and Spearman's rank, were applied to show the effectiveness of proposed system.

A supervised machine learning-based sentiment analysis system for analyzing student reviews about teacher's performance is proposed [9]. Support Vector Machine (SVM) is used for classifying reviews into positive, negative or neutral. The results show satisfactory performance of the proposed system with respect to comparing methods.

Different categories of emotions are detected and classified from textual responses regarding student feedback [10]. For this purpose, latent sentiment analysis and non-negative matrix factorization (NMF) are implemented, and it is reported that NMF model performed better. However, better results can be achieved by extending-student feedback corpora.

Instead of using traditional questionnaire-based feedback evaluation, an automatic sentimentbased performance evaluation system is proposed by [11]. The supervised and semi-supervised machine learning approaches are used, supported by feature identification and computation module. The results show that the Naïve Bayes algorithm performed better by achieving an accuracy of $90 \%$. However, using machine learning algorithm like SVM and Neural Network, along with specific knowledge can produce better performance.

A student feedback system is proposed [12] using different text analysis techniques, such as topic extinction through clustering, sentiment classification via link pipe, and summarization using $\mathbf{J}$ Free Charts . It is reported that clustering along with cosine similarities is efficient for topic extraction and logistic regression performed better for sentiment classification.

A faculty rating system based on student feedback is proposed by [13]. For this purpose, the Naïve Bayes classification algorithm was implemented, and faculty was classified into different classes 
based on five-star rating. Further improvement can be made by increasing the size of data set and applying other classifiers.

A conceptual framework for gathering and analyzing student sentiment using text analysis techniques such as preprocessing, sentiment abstraction and feedback summarization is proposed by [14]. To evaluate of effectiveness of the proposed framework, a case study was conducted by selecting courses from school of information system.

To analyze student feedback in real time mode, [15] proposed an automatic sentiment-base feedback analysis system using different machine learning techniques, supported by different combination of features and preprocessing steps. The results obtained show that Support Vector Machine (SVM) has achieved the highest accuracy of $95 \%$.

A voting and symbol method based on machine learning is proposed by [16] for evaluating performance of teaching faculty. For this purpose, different machine learning algorithm, such as Naïve Bayes, Decision tree, J48 and ID3 were used. The results depict that voting and symbol method combined with Chi-Square has given improved performance than the other methods.

The work proposed by [17] used different machine learning algorithm, such as K-Nearest Neighbor and Naïve Bayes with bag of words and TF-IDF computation for classifying student feedback comments regarding the under graduate courses. A small data set of student feedback about course evaluation was used, which was one of the major limitation of their work.

A sentiment classification for analyzing student feedback collected from Facebook and Twitter regarding their teachers is proposed by [18]. For this purpose, two machine learning algorithms, namely Naïve Bayes and Support Vector Machine were used. Results show that both classifications provided satisfactory results.

A lexicon-based approach is used to analyze student textual feedback for predicting performance of teaching faculty [19]. The method is based on a manually created lexicon containing sentiment words and intensifiers. The result was presented in a way showing sentiments of student at different levels of granularities. 
An emotion detection system in E-learning domain is proposed by [20]. The system was capable of classifying student opinions regarding learning progress. Gate software was used to implement the frame work.

\section{Methodology}

Firstly, we present a few baseline techniques and then present the proposed technique. In all such techniques, either lexicon is used or a feature vector is created for a given tweet, which is applied as its feature set with the classifiers.

Baseline Methods: We perform experiments with three baseline methods, namely: (i) lexiconbased SA for student feedback analysis [2], (ii) fuzzy-based student feedback analysis [5], and (iii) Supervised machine learning approach for teacher's performance evaluation [3]. The first study [2] has used lexicon for sentiment scoring of opinion words only, whereas, we have proposed improved sentiment scoring technique for both opinion words and modifiers used in student feedback. The $2^{\text {nd }}$ baseline study [5] has used traditional questionnaire-based data collection and analysis, whereas our proposed work uses sentiment-based approach in which aggregated sentiment score is made input to fuzzy logic module for analyzing student feedback and satisfaction. Finally, a machine learning technique are applied by [3] for student feedback analysis based on classical feature sets.

\section{Proposed Method}

We investigate Fuzzy-based sentiment analysis for analyzing student feedback and satisfaction inspired by Ghani et al. [4] work on applying fuzzy-based SA for measuring customer loyalty, we leverage fuzzy-based SA for analyzing student feedback and satisfaction.

The proposed system (Fig. 1) employs a fuzzy-based SA approach for analyzing student feedback in terms of classifying opinion and emotion words expressed in the feedback comments. The proposed system is beneficial for education sector with respect to automated analysis of student comments/feedback about the teaching faculty for improving the quality of faculty. 


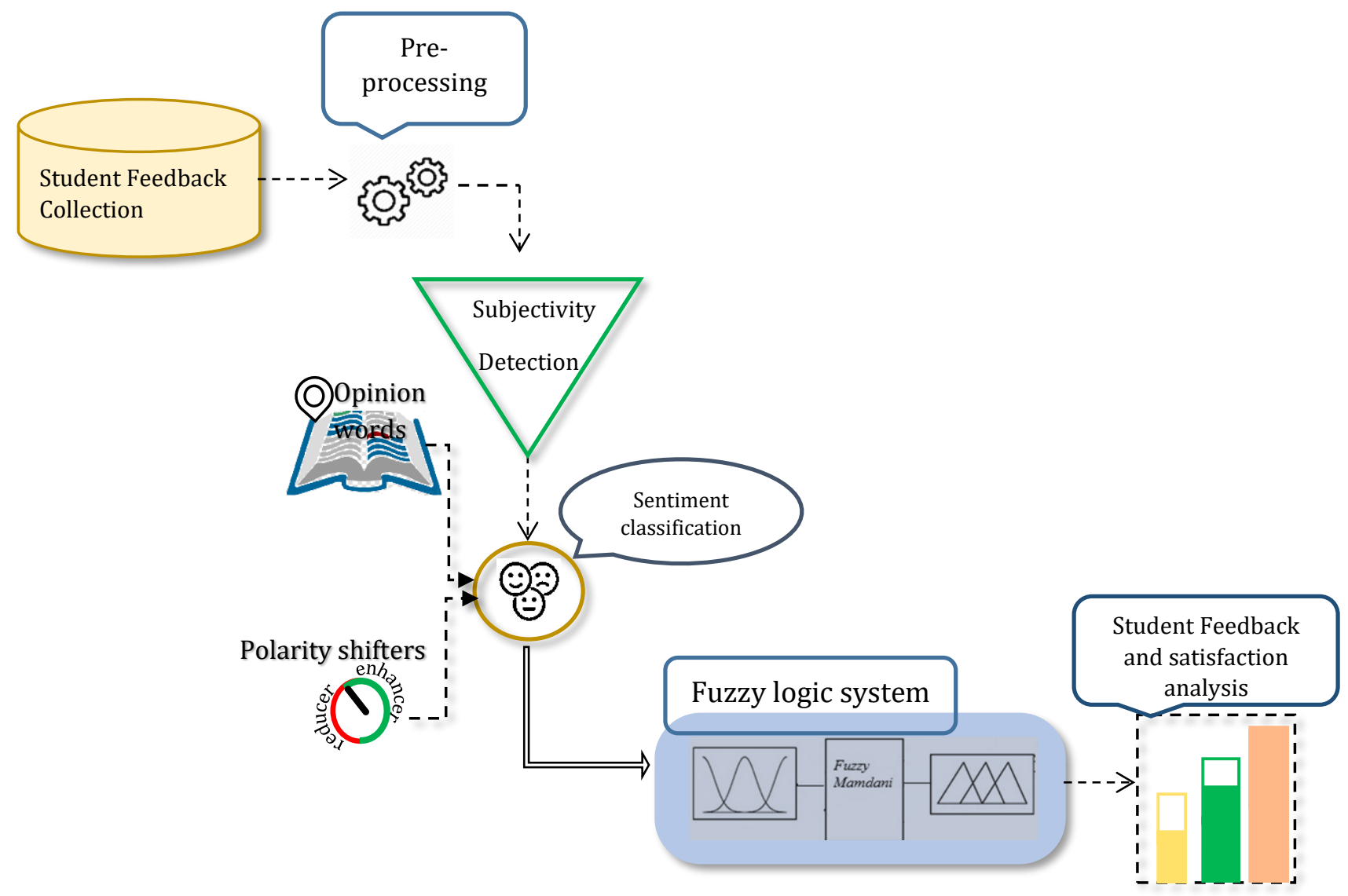

Fig. 1 Proposed System 


\subsection{Lexical Resources Used}

We used following lexical resources in the proposed work.

\subsubsection{Opinion Lexicon}

The opinion lexicon [21], contains more than six thousand +ive and -ive opinion words. A sample list of positive and negative opinion words is presented in Table 1 and Table 2.

Table 1 A partial list of positive opinion words

\begin{tabular}{|c|c|c|c|}
\hline Affection & Satisfactory & Marvellous & Affordable \\
\hline Courage & Favourite & Peaceful & Believable \\
\hline Progress & Fearless & Effective & Gorgeous \\
\hline Regard & Efficient & Favour & Feasible \\
\hline
\end{tabular}

Table 2 A partial list of negative opinion words

\begin{tabular}{|l|l|l|l|}
\hline Injustice & Insensitivity & Sadness & Wrong \\
\hline Loud & Conflict & Shameful & Lack \\
\hline Foolish & Aggression & Violent & Lose \\
\hline Mysterious & Forbidden & Disagree & Unfaithful \\
\hline
\end{tabular}

\subsubsection{SentiWordNet}

The SentiWordNet (SWN) lexicon contains more than sixty thousand entries, used for assigning sentiment scores to opinion words appearing in the student feedback text. For example, in text " $I$ am very impressed from his teaching method", the word "very" depicts polarity shifter and the word "impressed" is an opinion word. Each word in SWN has three sentiment scores: +ive, -ive and neutral, having values between 0 and 1. In Table 3, a sample word from SWN is presented, having POS tag, sense-id, synsets and gloss terms. 
Table 3 SentiWordNet sample entry of word "comfortable"

\begin{tabular}{|c|c|c|c|c|c|c|c|}
\hline Term & $\begin{array}{c}\text { POS_ }_{-} \\
\text {Tag }\end{array}$ & $\begin{array}{c}\text { Sense } \\
\text { ID }\end{array}$ & $\begin{array}{c}\text { Pos_ }_{-} \\
\text {Score }\end{array}$ & $\begin{array}{c}\text { Neg_ }_{-} \\
\text {Score }\end{array}$ & $\begin{array}{l}\text { Neu_ }_{-} \\
\text {Score }\end{array}$ & Synset & Gloss \\
\hline $\begin{array}{l}\text { Comfor } \\
\text { table }\end{array}$ & A & 479330 & 0.625 & 0 & 0.375 & $\begin{array}{l}\text { comfortable\#1 } \\
\text { comfortable\#2 } \\
\text { comfortable\#3 } \\
\text { comfortable\#4 } \\
\text { comfortable\#5 }\end{array}$ & $\begin{array}{l}\text { free from stress or conducive } \\
\text { to mental ease; "was settled in } \\
\text { a comfortable job, one for } \\
\text { which he was well prepared" }\end{array}$ \\
\hline
\end{tabular}

\subsection{Student Feedback Collection}

The student feedback data is collected from different feedback sites such as [22, 23]. These public datasets contain teacher evaluation conducted in different universities. Table 4 shows detail of the acquired dataset.

Table 4 Detail of dataset

\begin{tabular}{|l|l|l|l|l|}
\hline Dataset & Description & $\begin{array}{l}\text { Total no. of } \\
\text { reviews }\end{array}$ & $\begin{array}{l}\text { No of positive } \\
\text { reviews }\end{array}$ & $\begin{array}{l}\text { No. of negative } \\
\text { rev }\end{array}$ \\
\hline D1 & $\begin{array}{l}\text { Student } \\
\text { feedback }\end{array}$ & 1415 & 955 & 249 \\
\hline
\end{tabular}

\subsection{Preprocessing}

This module is applied to remove noise from acquired dataset by applying different preprocessing steps, such as sentence and word tokenization, stop word removal, stemming, lemmatization and spell correction [24].

Tokenization: Python-based NLTK tokenizer is used for segmenting the text into small parts, called tokens.

Stop word removal: NLTK has stop-word corpus which comprises of stop-word list for many languages. So, in the next step, tokenized text is further processed by removing stop-words by using Python NLTK.

Case conversion: In case conversion, the uppercase words in the feedback are changed into lower case. For example, a word "WOW", is change to "wow". 
Spelling correction: This module is used to correct spelling mistakes committed by the user while typing review sentences. The python-based library, namely A spell is used for this [25].

\subsection{Sentiment Classification}

This module performs two basic operations: (i) subjectivity detection, and (ii) sentiment classification. An overview of each module is presented as follows.

\subsubsection{Subjectivity Detection}

In this phase, input text from student feedback is classified as subjective or objective using different opinion lexicons. The objective text contains no opinion words, whereas the subjective text includes opinionated terms [26].

The subjectivity detection classification module aims at identifying and retaining subjective words, phrases, and tweets by checking their existence in a number of opinion lexicons. For a given review, each sentence is scanned for checking the existence of opinion terms with the help of different opinion lexicons.

A sentence having one/more opinion words is labelled as subjective, otherwise it is declared as objective tweet. For example, in tweet: "The teaching style is amazing", the word "beautiful" is an opinion term, and therefore, we mark this tweet as subjective using Eq. (1).

$$
\text { Tweet }_{\text {sub_obj }}=\left\{\begin{array}{cl}
\text { subjective, } & \text { if }\left(\left(w_{\mathrm{x}} \in \mathrm{OL}\right)\right) \\
\text { objective, } & \text { if }\left(\mathrm{w}_{\mathrm{x}} \notin \mathrm{OL}\right) \mathrm{V}
\end{array}\right.
$$

Where, $W_{x}$ is a word in given tweet, OL is an opinion lexicon,

The proposed subjectivity detection module (Fig. 2) eliminates non-opinion terms, while retaining the opinion terms, and resultantly, time and effort required for calculating scores of words in subsequent sub-modules also reduces.

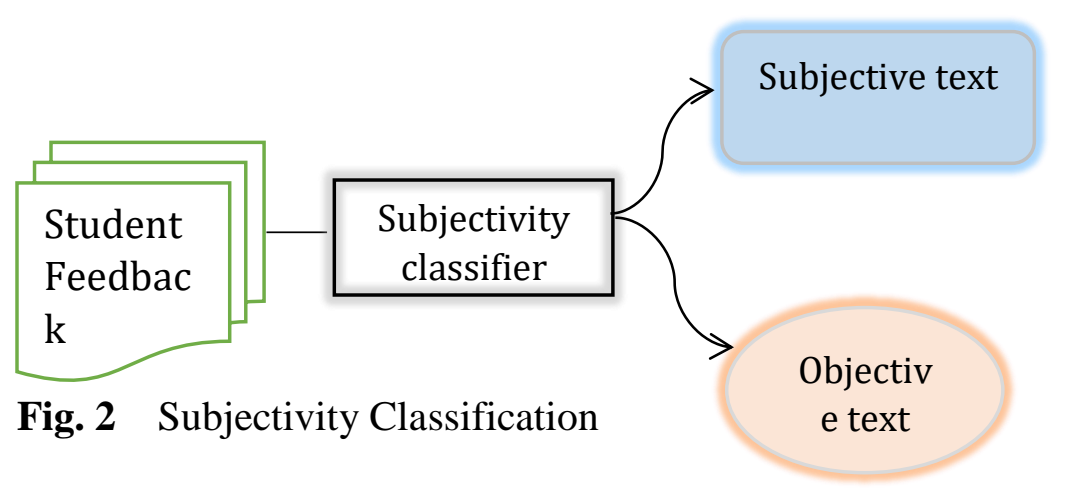


Table 5 shows a sample list of tweets labeled as subjective or objective by the subjectivity detector module. The tweet is tagged as subjective, if it carries one/more opinion words. For example, tweet\#1 is labeled as subjective due to presence of opinion words: "love" and "enjoy",

Table 5 Example tweets with subjectivity detection

\begin{tabular}{|l|l|l|l|}
\hline Tweet\# & Review Tweet & Opinion Word(s) & Subjective/Objective \\
\hline 1 & The teaching style is excellent & "excellent" & Subjective \\
\hline 2 & My teacher encourages me & "encourage", & Subjective \\
\hline 3 & $\begin{array}{l}\text { Abdullah is performing } \\
\text { classroom tasks }\end{array}$ & -------- & Objective \\
\hline 4 & $\begin{array}{l}\text { Aziz cooperates consistently } \\
\text { with the other colleague and } \\
\text { other students }\end{array}$ & $\begin{array}{l}\text { "cooperates", } \\
\text { "consistently" }\end{array}$ & Subjective \\
\hline 5 & $\begin{array}{l}\text { My teacher is confident, and a } \\
\text { great role model for us }\end{array}$ & $\begin{array}{l}\text { "confident", } \\
\text { "positive", }, \text { great", } \\
\text { role model" }\end{array}$ & Subjective \\
\hline
\end{tabular}

\subsubsection{Sentiment Classification}

In this step a lexicon-based sentiment classification technique is applied by computing sentiment scores of opinion words and polarity shifters. This module is an extension of the work proposed by [2] by revising the sentiment scoring technique for opinion words, and polarity shifters to efficiently classify the student's feedback. They used word frequency and word polarity, whereas, we propose to assign sentiment scores to both opinion words and modifiers using lexicon-based technique, which resulted in improved performance.

\subsubsection{Sentiment classification of opinion words in Student Feedback}

The sentiment classification of opinion words is performed using Sent WordNet scoring technique [27] as follows:

According to the SWN structure, each word have more than one senses. So, we consider three sentiment scores: +ive, -ive and neutral for multiple senses in SWN [24] to decide accurate sense 
for a sentiment word. Using Eq. 2, Eq. 3, and Eq. 4, we take an aggregate of sentiment score for each of +ive, -ive and neutral words.

$$
\begin{gathered}
A g r_{-} p o s\left(w_{j}\right)=\sum_{j=0}^{n} p o s_{-} w(j) \\
A g r_{-} n e g\left(w_{j}\right)=\sum_{j=0}^{n} n e g_{-} w(j) \\
A g r_{-} n e u\left(w_{j}\right)=\sum_{j=0}^{n} n e u_{-} w(j)
\end{gathered}
$$

In the next step, Eq. 5, Eq. 6, and Eq. 7 are used to calculate the positive, negative and neutral words average sentiment scores that is given as follows;

$$
\begin{gathered}
P_{\text {avg }}\left(w_{j}\right)=\operatorname{Agr\_ pos}\left(w_{j}\right) / \operatorname{totalsyn}\left(w_{j}\right) \quad j=1 \ldots n \\
N_{\text {avg }}\left(w_{j}\right)=\operatorname{Agr\_ neg}\left(w_{j}\right) / \operatorname{totalsyn}\left(w_{j}\right) \\
\operatorname{Neu}_{\text {avg }}\left(w_{j}\right)=1 \ldots n \\
\operatorname{Agr\_ neu}\left(w_{j}\right) / \operatorname{totalsyn}\left(w_{j}\right) \\
\end{gathered}
$$

Where, the aggregate sentiment scores of the $j t h+i v e,-i v e$ and neutral synset of a word $w_{j}$, is denoted by $A g r_{-} p o s\left(w_{j}\right), A g r_{-} n e g\left(w_{j}\right)$, and $A g r_{-} n e u(w \mathrm{j})$ and totalsyn is the total number of synsets for the word $w_{j}$.

Consider the example text shown in Table 6.

Table 6 Example Text with part-of-speech tagging and sentiment word

\begin{tabular}{|l|l|}
\hline Example text & Long vacations. \\
\hline POS tagged text & Long/JJ vacations/NN \\
\hline Sentiment word & Long \\
\hline
\end{tabular}


In Table 6 the part of speech tag for sentiment term (“long”) is adjective (JJ), whereas in SWN, there are 12 senses for the word "long", 1 for verb, 2 for adverb and 9 senses for adjective. Applying Eq. 5, Eq. 6 and Eq. 7, the average +ive, -ive and neutral scores for 9 senses of adjective, are calculated as follows:

$$
\begin{gathered}
P s_{\text {Score }}(\text { long })=\frac{(0.125+0+0+0+0+0.250+0.375+0.00+0.250)}{9}=0.111 \\
N g_{\text {score }}(\text { long })=\frac{(0.375+0+0+0+0+0+0.5+0.5+0.12)}{9}=0.167 \\
N e u_{\text {Score }}(\text { long })=\frac{(0.5+1+1+1+1+0.75+0.875+0.5+0.63)}{9}=0.722
\end{gathered}
$$

It is obvious from above computations, that $0.111,0.167$ and 0.722 are the average +ive, -ive and neutral sentiment scores of all senses of word "long" respectively.

The final sentiment score is computed by choosing the dominant sentiment score of an opinion word "opw", as follows:

sen_score $($ opw $)$

$$
=\left\{\begin{array}{cc}
P s_{\text {Score }} & \text { if } \max \left(P s_{\text {Score }}, N g_{\text {score }}, N e u_{\text {Score }}\right)=P s_{\text {Score }} \\
N g_{\text {Score }} & \text { if } \max \left(P s_{\text {Score }}, N g_{\text {Score }}, N e u_{\text {Score }}\right)=N g_{\text {Score }} \\
\text { else }
\end{array}\right.
$$

The sen_score $(o p w)$ is +ive, if the mean +ive score $\left(P s_{S c o r e}\right)$ is larger than the mean -ive $\left(N g_{\text {score }}\right)$, and neutral neu $\left.u_{\text {score }}\right)$ score, else -ive. The sentiment score is neutral $\left(\right.$ neu $\left.u_{\text {Score }}\right)$, if the mean +ive and -ive sentiment scores are identical or the neutral sentiment score is larger than the +ive and -ive. In the aforementioned case, the sentiment scores $\left(P s_{\text {Score }}, N g_{\text {Score }}, N e u_{\text {Score }}\right)$ for the word "long" are $\{0.111,0.167,0.722\}$. The dominant polarity score , is: sen_score("long")="0.722". 
In Table 7 a sample list of opinion words is shown.

Table 7 A sample list of opinion words with sentiment scores

\begin{tabular}{|l|l|l|}
\hline \multicolumn{1}{|c|}{$\begin{array}{c}\text { Opinion } \\
\text { Word }\end{array}$} & \multicolumn{1}{|c|}{$\begin{array}{c}\text { Sentiment } \\
\text { Score }\end{array}$} & \\
\hline Faithfully & $(+0.25)$ & Score assigned SWN-based scoring scheme (Eq. 3.1) \\
\hline Focused & $(+0.125)$ & Score assigned SWN-based scoring scheme (Eq. 3.1) \\
\hline Independently & $(-0.375)$ & Score assigned SWN-based scoring scheme (Eq. 3.1) \\
\hline Organised & $(-0.125)$ & Score assigned SWN-based scoring scheme (Eq. 3.1) \\
\hline Hesitate & $(+0.25)$ & Score assigned SWN-based scoring scheme (Eq. 3.1) \\
\hline Unsatisfied & $(-0.875)$ & Score assigned SWN-based scoring scheme (Eq. 3.1) \\
\hline
\end{tabular}

\subsubsection{Sentiment Scoring of Polarity Shifters}

Intensifiers are the terms, which increase or decrease the intensity of opinion words in a sentence. For example, "very", "somewhat", "slightly", "too", "really", "extremely" etc., increase or decrease the semantic orientation of opinion word.

In this work, we used 50 English intensifiers proposed by [28]. We assigned a polarity score to each intensifier by using the numeric values (e.g. 1, -1, 0.5, -0.5), proposed by [27] to compile a list of +ive and -ive intensifiers (Table 8).

Let list_pol_shft is a list of positive and negative intensifiers represented as:

$$
\text { list_pol_shft }=\{\text { list of positive and negative intensifiers }\}
$$

If a term is present in a list of +ive or -ive intensifiers, then the sentiment score of the neighboring opinion word is calculated as follows:

$$
\begin{aligned}
& \text { sentiment } \text { score_pol_shifter }_{\text {opw }} \text { ) }
\end{aligned}
$$

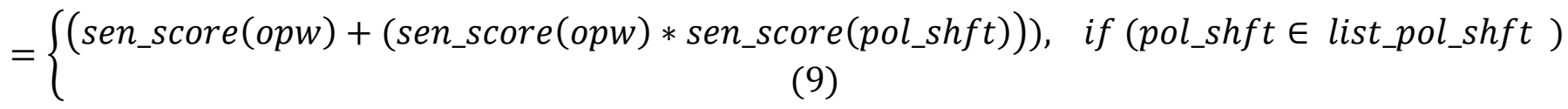


where, the word pol_shft represents a word belonging to a list of polarity shifters, opw is an opinion word, sen_score(pol_shft) is a sentiment score of the polarity shifter obtained from polarity shifter list list_pol_shft. The polarity score of nearest sentiment word is calculated by multiplying the sentiment score of polarity shifter by the sentiment score of an opinion word (using Eq. 9).

Table 8 Polarity shifters

\begin{tabular}{|l|l|l|l|l|l|}
\hline Intensifier & Score & Intensifier & Score & Intensifier & Score \\
\hline Too & $+45 \%$ & Totally & $+70 \%$ & Extremely & $+80 \%$ \\
\hline Pretty & $+20 \%$ & Less & $-50 \%$ & Very & $+50 \%$ \\
\hline Quite & $-20 \%$ & Hardly & $-70 \%$ & Slight & $-40 \%$ \\
\hline Completely & $+100 \%$ & Really & $+15 \%$ & & \\
\hline
\end{tabular}

\subsection{Applying an Example Student Feedback Review on Proposed Model}

An example of student feedback is as given as follows: <feedback> "the lecture was quite bad. $i$ am really unsatisfied with it.".

Taking the first sentence: To perform the sentiment classification on given feedback, we first use the sentiment classification of opinion words (section 3.3) in the first sentence. The sentiment score of the opinion word "bad" is computed as " -0.625 ", which is negative. Similarly, the sentiment score of the polarity shifter "quite" is $-20 \%$. Using polarity score of modifier and its associated opinion term is computed using Eq. 9 as follows:

$$
\begin{aligned}
& \text { sentiment }_{\text {score_pol_shifter }}(\text { "quite bad") } \\
& \quad=\left\{\left(\text { sen_score }(" b a d ")+\left(\text { sen_score }(" b a d ") * \operatorname{sen} \_s c o r e(" q u i t e ")\right)\right)\right.
\end{aligned}
$$

$=-0.625+[-0.625 *(-20 \%)]=0.45$ Here, the opinion word "helpful" is available in the SWN, its score is 0.25 , and the enhancer modifier "extremely" has weightage of -0.2. Therefore, we received a revised score of -0.5 . 
Taking the second Sentence: We take the 2nd sentence of feedback as: "i am really unsatisfied with it." In this sentence, sentiment score of opinion word "unsatisfied" is computed as "-0.875 ", which is negative. Similarly, the sentiment score of the polarity shifter "really" is "15\%". Using polarity score of modifier and its associated opinion term is computed using Eq. 9 as follows:

$$
\begin{aligned}
& \text { sentiment }_{\text {score_pol_shifter }}(\text { "really unsatisfied") } \\
& =\left\{\left(\text { sen_score }(\text { "unsatisfied" })+\left(\text { sen_score }(" \text { unsatisfied" }) * \operatorname{sen} \_ \text {score }(\text { "really") })\right)\right.\right.
\end{aligned}
$$

$=-0.875+[-0.875 * 15 \%]=-1$. Here, the opinion word "unsatisfied" is available in the SWN, its score is 0.875 , and the enhancer modifier "really" has weightage of 0.15 . Therefore, we received a revised score of -1 .

Table 9 shows sample sentences and their associated opinion words and enhancer.

\begin{tabular}{|c|c|c|c|c|c|}
\hline \multirow{2}{*}{$\begin{array}{l}\text { Sentence } \\
\text { id }\end{array}$} & \multirow[t]{2}{*}{ Input sentence } & \multicolumn{2}{|c|}{ Opinion words } & \multirow[b]{2}{*}{$\begin{array}{l}\text { Enhancer } \\
\text { Modifier }\end{array}$} & \multirow[b]{2}{*}{$\begin{array}{l}\text { Reducer } \\
\text { Modifier }\end{array}$} \\
\hline & & positive & Negative & & \\
\hline 1 & $\begin{array}{l}\text { the lecture was quite } \\
\text { bad. }\end{array}$ & - & $\begin{array}{l}\text { Bad } \\
(-0.625)\end{array}$ & - & $\begin{array}{l}\text { quite } \\
\text { (sentiment } \\
\text { score: } 0.2 \text { ) }\end{array}$ \\
\hline 2 & $\begin{array}{l}\text { i am really unsatisfied } \\
\text { with it. }\end{array}$ & - & $\begin{array}{l}\text { unsatisfied } \\
(-0.875)\end{array}$ & $\begin{array}{l}\text { really } \\
\text { (sentiment } \\
\text { score: }-0.15 \text { ) }\end{array}$ & -- \\
\hline
\end{tabular}

Table 9 Review text and its associated opinion words and modifier

\subsubsection{Computing Sentence-level Sentiment Score}

To compute the sentiment score of the entire feedback, the average score of all sentences is computed as follows:

$a v g_{\text {score }}=\frac{1}{n} \times \sum_{i=1}^{n} o p w_{r_{\text {score }}}\left(P_{\text {shifter }}\right)_{i}$ 
Where, "opw $w_{r_{\text {score }}}\left(P_{\text {shifter }}\right)_{i}$ " is the revised sentiment score of ith sentence (Eq. 10) and " $n$ " is the total no. of sentences in a given feedback. Taking the example review and putting the values in Eq. 10, we get:

$\operatorname{avg}_{\text {score }}=\frac{-0.5+(-1)}{2}=-0.75$

The value of " $a v g_{\text {score }}$ "calculated lies in the range $[-1,1]$. As it is necessary that the value of " $a v g_{\text {score }}$ " should be in between the $[0,1]$, so we normalize its value using min-max normalization; upon applying min-max normalization to " $a v g_{\text {score }}$ ", we get the normalized value as follows:

$\operatorname{avg}_{N}=\frac{\operatorname{avg}_{\text {score }}+1}{2}$

$\operatorname{avg}_{N}=\frac{-0.75+1}{2}=0.12$

Using aforementioned computations, we classify the student feedback as follows:

Student_feedback_sentiment_class $=$

$\left\{\begin{array}{c}\operatorname{Negative}(N), \text { if }\left(\operatorname{avg}_{N}>0 \text { and } \operatorname{avg}_{N} \leq 0.3\right) \\ \operatorname{Neutral}(N u), \text { else if }\left(\operatorname{avg}_{N}>0.3 \text { and } a v g_{N} \leq 0.6\right) \\ \text { Positive }(P), \text { else if }\left(\operatorname{avg}_{N}>0.6 \text { and } \operatorname{avg} g_{N} \leq 1\right)\end{array}\right.$

\subsection{Output of Sentiment Analysis}

The output of SA is the sentiment class assigned on the basis of Eq. 12. Putting the aforementioned value of $a v g_{N}$ computed using Eq. 11 (" $a v g_{N}=0.12$ ), where sentiment score is evaluated using Eq. 4, then we get the sentiment class as:

Student_feedback_sentiment_class $(c)=$ "Negative" 
Aforementioned sentiment class, i.e. "Negative" is the output of our proposed sentiment analysis system. In the next phase, we measure, student satisfaction by applying different steps of the fuzzy logic system as follows:

\subsection{Fuzzy-based system for student satisfaction level}

In this step, to quantify the student satisfaction level from a given student feedback a fuzzy logic system is used (Fig. 3). The used fuzzy logic system contains following steps.

i. $\quad$ Fuzzy sets: Determine the input and output linguistic variables and their associated terms.

ii. $\quad$ Fuzzification: The crisp input is converted to the fuzzy values using membership function.

iii. $\quad$ Construct the membership functions for the fuzzy sets

iv. $\quad$ Fuzzy if/then rules are constructed

v. Defuzzification: The fuzzy values are converted into the crisp values (non-fuzzy values).

Mathematically, the fuzzy logic system is describe in Eq. 13 as follows [4].

$$
A=\{x, u A(x)) \mid x \in X)
$$

where $\mu A(x)$ represent the membership function or degree of membership function, of $\mathrm{x}$ in $\mathrm{A}$ and $\mathrm{X}$, is the Universal set.

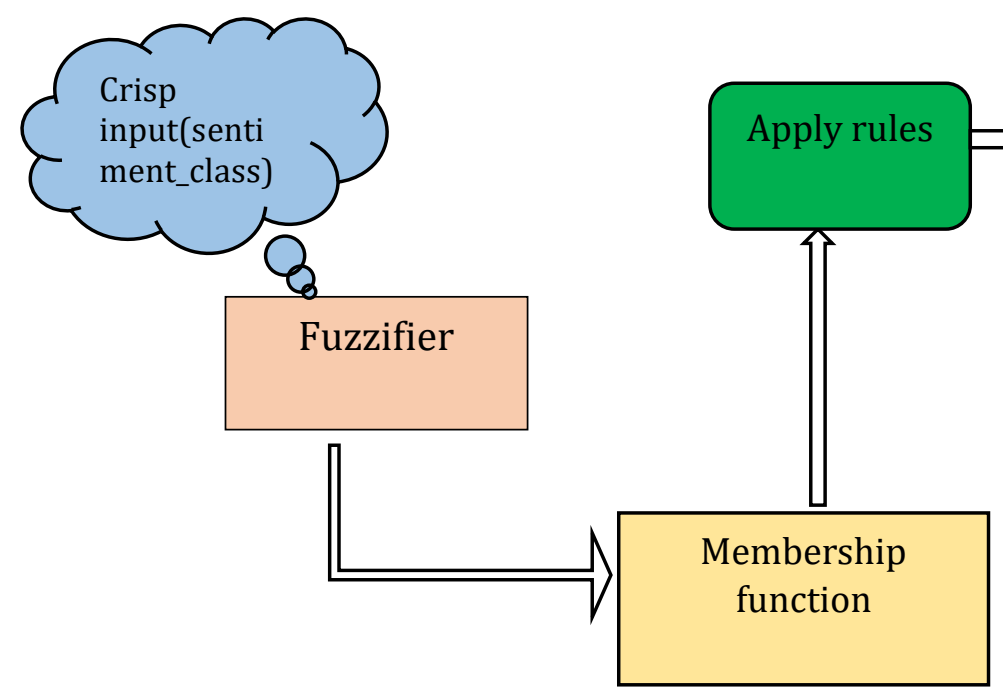


Fig. 3 Fuzzy logic based system

\subsubsection{Input and output Linguistic variables}

In our work, we take sentiment class as an input linguistic variable and student satisfaction as an output variable as shown in Table 10.

Table 10 Input and output linguistic variable.

\begin{tabular}{|l|l|}
\hline Type & Linguistic variable \\
\hline Input variable & Sentiment class \\
\hline Output variable & Student satisfaction \\
\hline
\end{tabular}

\subsubsection{Fuzzification}

In a fuzzy logic system, we first identify the input and output variables. In this step, to obtain the fuzzified values, the transformation of crisp input values into fuzzy set is performed and this process of conversion is known as fuzzification [29].

Based on the input-output linguistic variable, we determine their associated linguistic terms. We assigned three linguistic terms for the input variable and similarly, three linguistic terms have been taken for the output variable as shown in Table 11.

Table 11 Linguistic variable and Linguistic terms

\begin{tabular}{|c|c|}
\hline \multicolumn{2}{|l|}{ Input } \\
\hline $\begin{array}{l}\text { Linguistic } \\
\text { variable }\end{array}$ & Linguistic terms \\
\hline \multirow[t]{3}{*}{ Sentiment class } & Negative(N) \\
\hline & Neutral(Nu) \\
\hline & Positive(P) \\
\hline \multicolumn{2}{|l|}{ Output } \\
\hline $\begin{array}{l}\text { Linguistic } \\
\text { variable }\end{array}$ & Linguistic terms \\
\hline \multirow[t]{3}{*}{ Student satisfaction } & Not satisfied \\
\hline & Moderate \\
\hline & Satisfied \\
\hline
\end{tabular}




\subsubsection{Membership function}

The fuzzy logic system used membership function to plot the fuzzy sets. Different types of membership functions are available like Bell membership, triangular membership function, and Gaussian membership [30]. In our proposed work, we take riangular membership function which is describe in Fig 4.

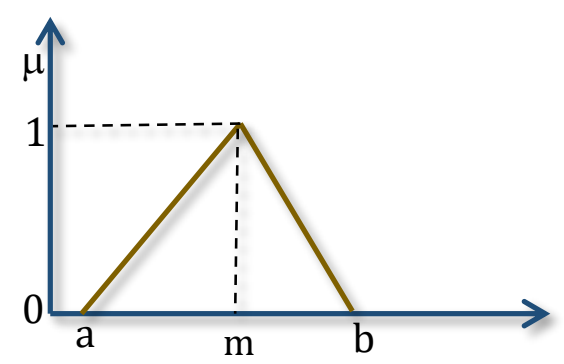

Fig. 4 Graphical representation of trimf.

The triangular membership function is defined by three parameters [a, b, c], where $a$ denotes lower boundary, $b$ is an upper boundary, 0 is the membership degree and $\mathrm{m}$ represents the center, where membership degree is 1 (Eq. 14).

$\operatorname{trimf}(x: a, b, m)=\left\{\begin{array}{rl}0 & x \leq a \\ \frac{x-a}{m-a} & a \leq x \leq m \\ \frac{b-x}{b-m} & m \leq x \leq b \\ 0 & c \leq x\end{array}\right.$

The membership functions for the linguistic terms of the sent_class variable is plotted as shown in Fig 5.

\begin{tabular}{|l|l|}
\hline Fuzzy sets & Membership function \\
\hline Negative & Triangular \\
\hline Neutral & Triangular \\
\hline Positive & Triangular \\
\hline
\end{tabular}




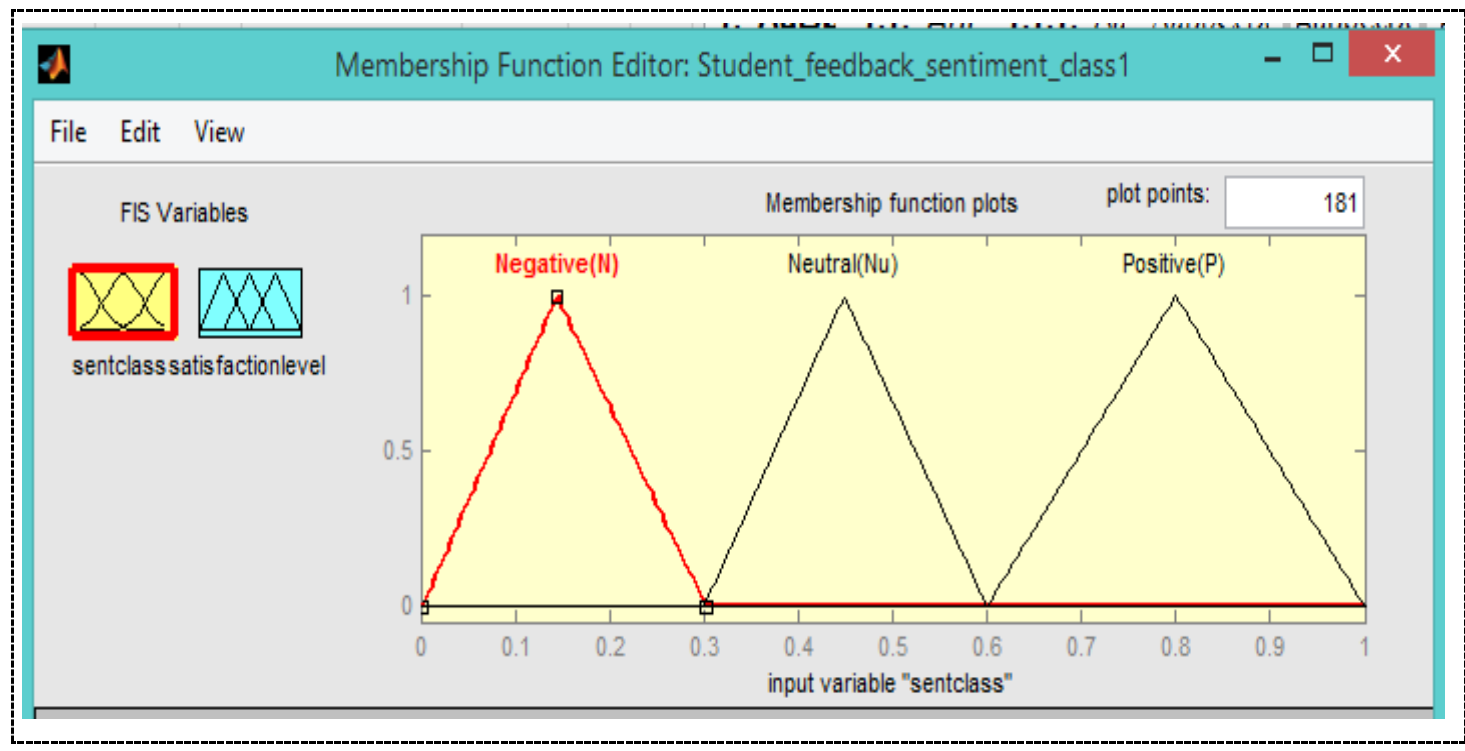

Fig. 5 Membership functions for sentiment class.

\subsubsection{Design if/then rules}

In this step, the fuzzy rules are designed to take the conclusion. These rules are simple and expressed as follow [31]:

\section{IF (antecedent) THEN (consequence)}

Where, If part is known as antecedent and then part is known consequent. Table 9 shows different fuzzy rules:

Table 12 Fuzzy Rules for student satisfaction.

\section{If/then Rules:-}

1. If(sentclass is negative) then (studentsatisfaction is Notsatisfied)

2. If(sentclass is neutral) then (customersatisfaction is moderate)

3. If(sentclass is positive) then (customersatisfaction is satisfied)

\subsubsection{Defuzzification}

Finally, to determine the student satisfaction, the defuzzifiction function is used in which fuzzy values are transformed in to the crisp values. In the proposed work, the Mamdani inference system is used, which computes the center of gravity using Eq. 15 as follows [4]; 
$Y=\int_{\min }^{\max } \mu(y) y d y / \int_{\min }^{\max } \mu(y) d y$

Where $\mathrm{Y}$ is the result of defuzzification, $\mu(\mathrm{y})$ is the membership function, $\mathrm{y}$ is the output variable, min is the lower limit, and max is the maximum limit for defuzzification.

In Fig. 6, the membership function for the output variable student satisfaction, is plotted.

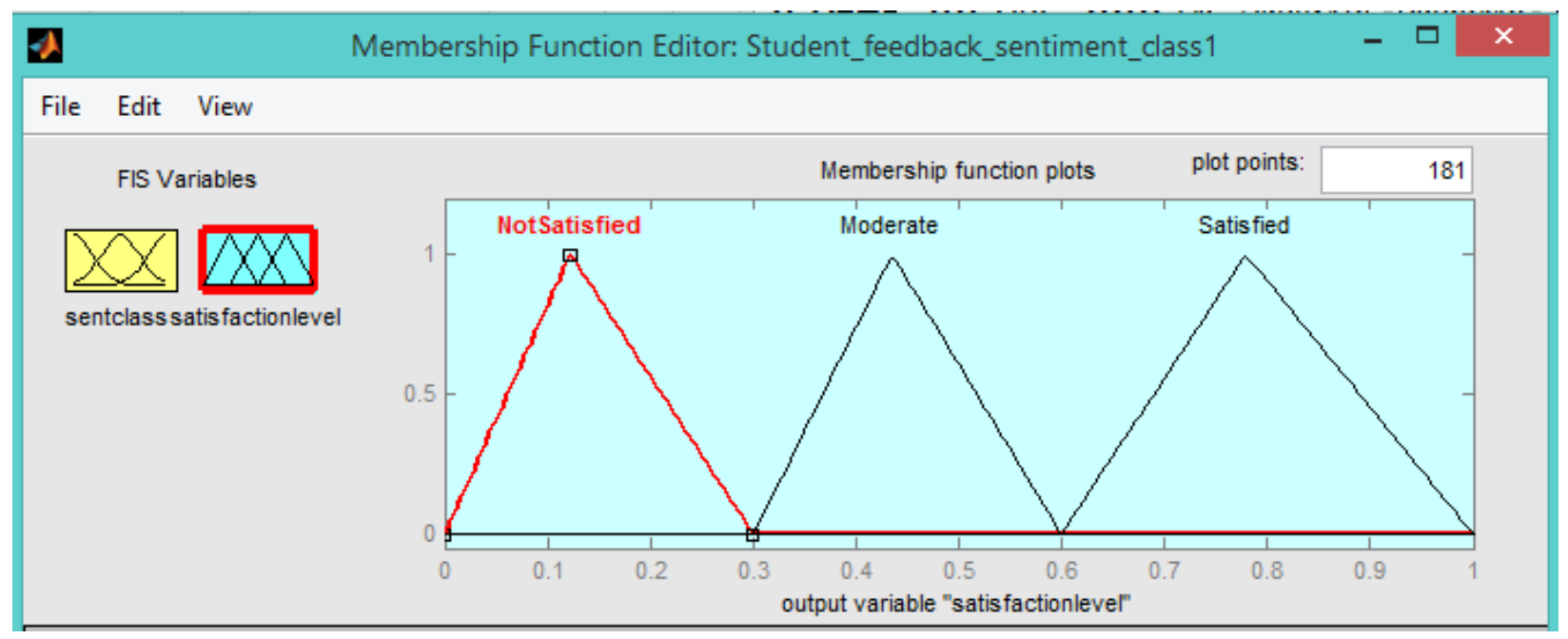

Fig. 6 Membership functions for output variable student satisfaction

To measure student satisfaction with the sentiment class, we simulate the rules in MATLAB. For instance, in Fig. 7, if the sentiment score is 0.12 , which is considered as negative, then the student satisfaction is 0.141 , considered as not-satisfied and very close to the sentiment score. 


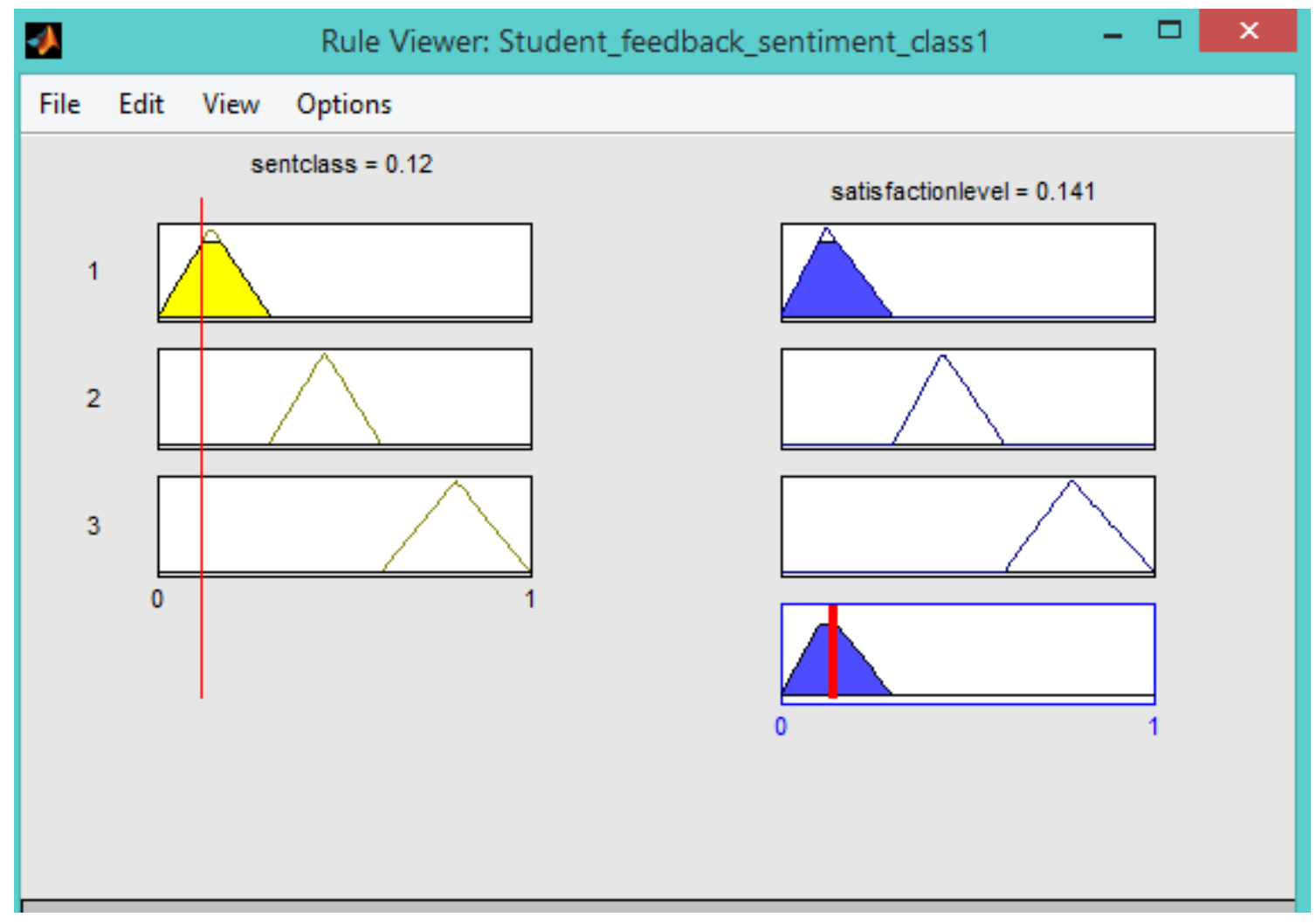

Fig. 7 Matlab Rule Viewer.

For the given input student review "the lecture was quite bad. $i$ am really unsatisfied with it, we have reached to the conclusion that student satisfaction level is "not-satisfied", which means student is not-satisfied on the basis of example feedback, presented in section 3.3.2.

The pseudo code steps for the development of the proposed system is presented in Algorithm 1.

Algorithm 1 Algorithm for efficient classification of sentiment and measuring student satisfaction level.

Input: Student feedback

Output: Student satisfaction level \#Retrieve “dataset" feedback

1. Student Feedback collection

2. Preprocessing

3. Subjectivity detection(S) (Eq. 1) 
4. for (Opw in S AND polarity_shifter in S)

5. if $(\mathrm{Opw} \in \mathrm{SWN})$ then

6. Obtain Opw score from SWN (Eq. 5, Eq. 6, Eq. 7)

7. if (polarity_shifter $\in$ list_pol_shft) then

8. Obtain polarity_shifter score (Table 8)

9. end if

10. end for

11. Calculate final sentiment scoring (Eq. 12)

12. Assign sentiment as positive, neutral, and negative. \#Use fuzzy logic system.

13. Perform Fuzzification on sentiment classes (Eq. 13)

14. Apply if/then rules (Table 9)

15. Defuzzification (Eq.15)

16. Output the student satisfaction level as 'dissatisfied', 'neutral', and 'satisfied'.

\section{Result and discussion}

In this chapter, results are analyzed on account of conducting experiments and answers are given to the posed research questions.

\subsection{Experiment \#1}

Answer to RQ1: To answer this research question, "How can we perform efficient classification of opinion words expressed by student in their feedback by revising the sentiment scoring technique proposed in baseline method?", we applied a fuzzy-based technique for the sentiment classification of student feedback (technique discussed in methodology). Additionally, we conducted experiment on the state of art machine learning classifier namely Naïve Bayesian (NB), Random Forest(RF), Support Vector Machine(SVM).

Table 13 shows result on account of applying the aforementioned machine learning classifiers and it is evident the proposed model performed better. The basic reason behind the efficient classification of proposed model with respect to Machine Learning(ML) classifiers is that the both 
opinion word and polarity shifters are detected and assigned proper sentiment scores, and resultantly our proposed system efficiently classified the input text in the form of student feedback.

Table 13 Comparison with machine learning classifiers

\begin{tabular}{|l|l|l|l|l|}
\hline ML classifiers & P & R & F & A \\
\hline NB & 0.82 & 0.79 & 0.72 & 0.78 \\
\hline RF & 0.85 & 0.85 & 0.84 & 0.85 \\
\hline SVM & 0.78 & 0.79 & 0.78 & 0.78 \\
\hline $\begin{array}{l}\text { Proposed } \\
\text { (Our work) }\end{array}$ & $\mathbf{0 . 8 7}$ & $\mathbf{0 . 9 7}$ & $\mathbf{0 . 9 0}$ & $\mathbf{0 . 8 9}$ \\
\hline
\end{tabular}

\subsection{Experiment \#2}

Answer to RQ2: “What is the efficiency of fuzzy-based student feedback sentiment classification system with and without considering polarity shifters?,?"

In second experiment, we evaluated the performance of proposed system with and without considering polarity shifters. While considering polarity shifters, Table 14 shows that, the proposed system performed slightly better in terms of Accuracy(A), Recall(R), F-measure(F). However, Precision $(\mathrm{P})$ results are identical in both the cases, i.e. "with polarity shifters" and "without polarity shifters".

Table 14 Comparative results with and without polarity shifters

\begin{tabular}{|l|l|l|l|l|}
\hline & A & P & R & F \\
\hline $\begin{array}{l}\text { With polarity } \\
\text { shifters }\end{array}$ & 0.85 & 0.87 & 0.97 & 0.94 \\
\hline $\begin{array}{l}\text { Without polarity } \\
\text { shifters }\end{array}$ & 0.81 & 0.83 & 0.95 & 0.89 \\
\hline
\end{tabular}




\subsection{Experiment \#3}

Answer to RQ3: “What is the efficiency of fuzzy-based sentiment analysis of student feedback using opinion words and polarity shifters w.r.t to state-of-the-art work and different supervised Machine Learning Algorithms?"

To answer RQ3, performance of the proposed system is compared with that of baseline studies [2, 3 , 5] using different performance evaluation metrics, namely precision, recall, f-measure, and accuracy. Result presented in the Table 15, show that the proposed model outperformed the baseline work $[2,3,5]$ in terms of improved precision and f-measure. The recall of the two systems is same. However, the precision, f-score and accuracy of the proposed system is higher than the baseline works.

Table 15 Comparison with baseline studies

\begin{tabular}{|l|l|l|l|l|l|}
\hline Study & Method & Precision(\%) & Recall(\%) & F-score(\%) & Accuracy(\%) \\
\hline Rajput et al. [2] & Lexicon-based & 0.40 & 0.97 & 0.57 & 0.79 \\
\hline Nasim et al. [3] & supervised & 0.79 & 0.82 & 0.80 & 0.82 \\
\hline $\begin{array}{l}\text { Yousif, et al. } \\
\text { [5] }\end{array}$ & $\begin{array}{l}\text { Classical } \\
\text { Fuzzy-based }\end{array}$ & 0.81 & 0.83 & 0.81 & 0.84 \\
\hline Proposed & $\begin{array}{l}\text { Sentiment } \\
\text { Driven Fuzzy- } \\
\text { based } \\
\text { sentiment } \\
\text { analysis }\end{array}$ & 0.89 & 0.97 & 0.90 & 0.94 \\
\hline
\end{tabular}

\subsection{Evaluating Student Satisfaction Level}

We plot a chart for measuring the efficacy of the proposed fuzzy-based model shown in Fig 8 . The $\mathrm{x}$ - axis denotes the student satisfaction level and the $\mathrm{y}$-axis denotes the sentiment score. Fig. 8 shows that if we have sentiment score of 0.4(Negative), then the customer satisfaction level is also 0.4(Not-satisfied). So it is observed that there is a direct relationship between sentiment score and student satisfaction level, i.e. if sentiment score is increasing, then customer satisfaction also increases. 


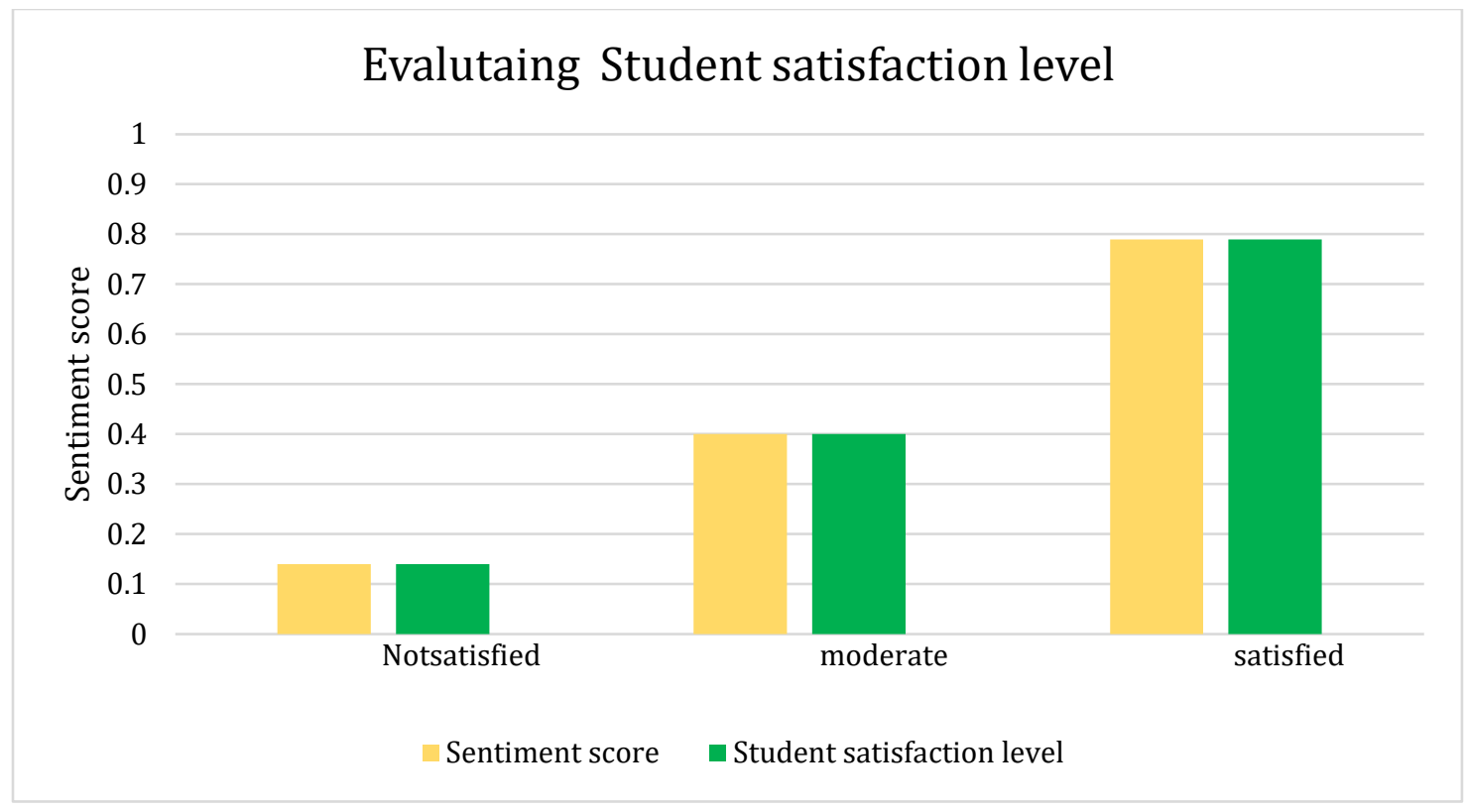

Fig. 8 Evaluating student satisfaction level with sentiment score.

\subsection{Statistical Analysis}

To investigate whether the proposed Fuzzy-based Sentiment Analysis for student satisfaction and feedback analysis with polarity shifters, is statistically significant than that of classical sentimentbased technique without polarity shifters and does not occur by chance, we conducted two experiments. From the dataset, we randomly choose 250 reviews in which each review is classified by both the proposed and classical sentiment-based techniques. The null and alternate hypothesis are formulated as follows"

Ho: Both the models have same error rate, and $\mathbf{H}_{\mathrm{A}}$ : Both models error rate is significantly different.

McNemar's test is computed as [32];

$$
\chi^{2}=\frac{\left(\left|m_{01}-m_{10}\right|-1\right)^{2}}{\left(m_{01}+m_{10}\right)}
$$

The significance results is presented in Table 16. 
Table 16 Performance Difference between the Proposed model (with polarity shifters) and Baseline (without polarity shifters) using significance test

\begin{tabular}{|c|c|c|c|}
\hline & & \multicolumn{2}{|c|}{$\begin{array}{l}\text { Baseline model [2] without polarity } \\
\text { shifters }\end{array}$} \\
\hline & & Correctly & Incorrectly \\
\hline & & Classified & Classified \\
\hline \multirow{2}{*}{$\begin{array}{l}\text { Proposed model (with } \\
\text { Polarity shifters) }\end{array}$} & Correctly Classified & 160 & 35 \\
\hline & Incorrectly Classified & 17 & 38 \\
\hline
\end{tabular}

The experiments are conducted to evaluate the performance of one of the baseline SA without using polarity shifters presented (Table 16). The baseline approach shows poor performance in terms of accuracy, precision, recall, and f-measure for student satisfaction analysis. However, the fuzzy-based approach with polarity shifters is significantly better than baseline method [2] with an accuracy of $82 \%$.

The statistical test validates that performance difference between that the proposed method (with polarity shifters) and the baseline method [2] (without polarity shifters) is statistically different. For 52 reviews, we can observe the discordant (Table 16) between the two models. i.e. the two models behave differently "with polarity shifters" and "without polarity shifters".

From the above discussion it is evident that the inclusion of polarity shifters significantly improved the performance of the proposed system for the fuzzy-based SA od student satisfaction and feedback analysis.

\section{Conclusion of future work}

The proposed system employs a fuzzy-based approach for the sentiment classification of student feedback by classifying opinion words and polarity shifters present in the student feedback comments. First of all, the student feedback data available as an open source, is preprocessed using different preprocessing techniques, such as stop word removal, tokenization, case conversion and spell correction. In the next step, sentiment classification of sentiment words and polarity shifters 
is carried out. An overall sentiment score is computed. Finally, the fuzzy logic system is applied to analyze customer feedback and satisfaction.

The experimental results are encouraging, and it is observed that the proposed system performed better than the baseline works and other state-of-the-art machine learning classifiers, in terms of accuracy, precision, recall, and f-measure.

\section{Limitations:}

The proposed approach has following deficiencies:

(1) Experimentation is performed on a data set with limited size, comprising of 1415 reviews collected as student feedback. This limited size of data set resulted in performance degradation of the system.

(2) The sentiment scoring of opinion words is based on the SentiWordNet (SWN) lexicon. The basic limitation of Senti WordNet (SWN) is lack of sufficient word coverage and certain words are not assigned a correct Senti WordNet (SWN) score.

(3) The proposed system cannot correctly classify certain opinion words and polarity shifters. For example, the input sentence: "He hardly late from the class" the modifier "hardly" treated as negative. The overall score and class became negative. However, the aforementioned sentence is actually giving positive sentiment. i.e. He is so punctual that he rarely comes late to class.

(4) One of the major limitation is associated with lexicon-based approach is that if a word or polarity shifter is not available in the given sentiment lexicon, then the system cannot correctly classify the student feedback.

(5) As it is era of social media like Facebook and Twitter, where students express their sentiments using emoticons and slang terms. However, the proposed system lacks the ability of classifying such construct (emoticons and slang terms).

\section{Future direction}

(1) The performance of the system can be improved by increasing the size of dataset. Furthermore, experimentation on multiple datasets needs to be carried out with respect to student feedback. 
(2) To assign more accurate sentiment score to opinion words, other sentiment lexicon, such as SentiFull and SenticNet needs to be investigated.

(3) To correctly classify opinion words and polarity shifters, corpus-base sentiment scoring technique needs to be experimented.

(4) It is required to conduct experiment using machine learning and deep learning techniques for efficient classification of student feedback by overcoming the "out-of-word" issue of a sentiment lexicon.

(5) To improve the performance of proposed system, emoticon lexicon and slang lexicon with proper sentiment scoring, needs to be investigated.

\section{Compliance with Ethical Standards:}

Funding: This study received no funding

Conflict of Interest: All authors declare that they have no conflict of interest

Ethical approval: This article does not contain any studies with human participants performed by any of the authors and does not contain any studies with animals performed by any of the authors.

Data Availability: The supplementary data used to support the findings of this study are available from the corresponding author upon request.

\section{References}

[1] Asghar, M. Z., Kundi, F. M., Ahmad, S., Khan, A., \& Khan, F. (2018). T-SAF: Twitter sentiment analysis framework using a hybrid classification scheme. Expert Systems, 35(1), e12233.

[2] Rajput, Q., Haider, S., \& Ghani, S. (2016). Lexicon-Based Sentiment Analysis of Teachers' Evaluation. Applied Computational Intelligence and Soft Computing, 2016 
[3] Nasim, Z., Rajput, Q., \& Haider, S. (2017, July). Sentiment analysis of student feedback using machine learning and lexicon based approaches. In Research and Innovation in Information Systems (ICRIIS), 2017 International Conference on (pp. 1-6). IEEE.

[4] Ghani, U., Bajwa, I., \& Ashfaq, A. (2018). A Fuzzy Logic Based Intelligent System for Measuring Customer Loyalty and Decision Making. Symmetry, 10(12), 761

[5] Yousif, M. K., \& Shaout, A. (2018). Fuzzy logic computational model for performance evaluation of Sudanese Universities and academic staff. Journal of King Saud UniversityComputer and Information Sciences, 30(1), 80-119

[6] Pavani, S., Gangadhar, P. V. S. S., \& Gulhare, K. K. (2012). Evaluation of teacher's performance using fuzzy logic techniques. International Journal of Computer Trends and Technology, 3(2), 200-205

[7] Jyothi, G., Parvathi, C., Srinivas, P., \& Althaf, S. (2014). Fuzzy expert model for evaluation of faculty performance in Technical educational Institutions. International Journal of Engineering Research and Applications, 4(5), 41-50

[8] Kaewyong, P., Sukprasert, A., Salim, N., \& Phang, A. (2015). The possibility of students' comments automatic interpret using lexicon based sentiment analysis to teacher evaluation. In 3rd International Conference on Artificial Intelligence and Computer Science (AICS2015) (pp. 179-189).

[9] Esparza, G. G., de-Luna, A., Zezzatti, A. O., Hernandez, A., Ponce, J., Álvarez, M., ... \& de Jesus Nava, J. (2017, June). A Sentiment Analysis Model to Analyze Students Reviews of Teacher Performance Using Support Vector Machines. In International Symposium on Distributed Computing and Artificial Intelligence (pp. 157-164). Springer, Cham.

[10] Mac Kim, S., \& Calvo, R. A. (2010, June). Sentiment analysis in student experiences of learning. In Educational Data Mining 2010.

[11] Kumar, A., \& Jain, R. (2015, October). Sentiment analysis and feedback evaluation. In MOOCs, Innovation and Technology in Education (MITE), 2015 IEEE 3rd International Conference on (pp. 433-436). IEEE.

[12] Nitin, G. I., Swapna, G., \& Shankararaman, V. (2015, October). Analyzing educational comments for topics and sentiments: A text analytics approach. In Frontiers in Education Conference (FIE), 2015 IEEE (pp. 1-9). IEEE. 
[13] Krishnaveni, K. S., Pai, R. R., \& Iyer, V. (2017, September). Faculty rating system based on student feedbacks using sentimental analysis. In Advances in Computing, Communications and Informatics (ICACCI), 2017 International Conference on (pp. 1648-1653). IEEE

[14] Gottipati, S., Shankararaman, V., \& Gan, S. (2017, October). A conceptual framework for analyzing students' feedback. In Frontiers in Education Conference (FIE) (pp. 1-8). IEEE.

[15] Altrabsheh, N., Cocea, M., \& Fallahkhair, S. (2014, November). Sentiment analysis: towards a tool for analysing real-time students feedback. In Tools with Artificial Intelligence (ICTAI), 2014 IEEE 26th International Conference on (pp. 419-423). IEEE.

[16] Pong-Inwong, C., \& Kaewmak, K. (2016, October). Improved sentiment analysis for teaching evaluation using feature selection and voting ensemble learning integration. In Computer and Communications (ICCC), 2016 2nd IEEE International Conference on (pp. 1222-1225). IEEE.

[17] Koufakou, A., Gosselin, J., \& Guo, D. (2016, July). Using data mining to extract knowledge from student evaluation comments in undergraduate courses. In Neural Networks (IJCNN), 2016 International Joint Conference on (pp. 3138-3142). IEEE

[18] Altrabsheh, N., Gaber, M., \& Cocea, M. (2013, June). SA-E: sentiment analysis for education. In International Conference on Intelligent Decision Technologies (Vol. 255, pp. 353-362).

[19] Aung, K. Z., \& Myo, N. N. (2017, May). Sentiment analysis of students' comment using lexicon based approach. In Computer and Information Science (ICIS), 2017 IEEE/ACIS 16th International Conference on (pp. 149-154). IEEE.

[20] Binali, H. H., Wu, C., \& Potdar, V. (2009, June). A new significant area: Emotion detection in e-learning using opinion mining techniques. In Digital Ecosystems and Technologies, 2009. DEST'09. 3rd IEEE International Conference on (pp. 259-264). IEEE.

[21] http://www.cs.uic.edu/ liub/FBS/sentiment-analysis.html\# lexicon

[22] www.ratemyprofessor.com

[23] www.myfaveteacher.com

[24] Asghar, M. Z., Khan, A., Ahmad, S., \& Kundi, F. M. (2013). Preprocessing in natural language processing. Editorial board, 152.

[25] (https://pypi.python.org/pypi/aspell-python-py2/1.1). 
[26] Asghar, M. Z., Khan, A., Zahra, S. R., Ahmad, S., \& Kundi, F. M. (2017). Aspect-based opinion mining framework using heuristic patterns. Cluster Computing, 1-19.]

[27] Asghar, M. Z., Khan, A., Ahmad, S., Qasim, M., \& Khan, I. A. (2017b). Lexiconenhanced sentiment analysis framework using rule-based classification scheme. PloS one, 12(2), e0171649.

[28] Full text of "Intensifiers in current English", available at:

https://archive.org/stream/intensifiersincu00benz/intensifiersincu00benz_djvu.txt, last accessed 10-nov-2018.

[29] Wang, X., Zhang, H., \& Xu, Z. (2016). Public sentiments analysis based on fuzzy logic for text. International Journal of Software Engineering and Knowledge Engineering, 26(09n10), 1341-1360.

[30] Alam, J., \& Pandey, M. K. (2017). A soft computing model for evaluating teachers' overall performance using fuzzy logic. Journal of Information Technology \& Software Engineering, 7(02).

[31] Darestani, A. Y., \& Jahromi, A. E. (2009). Measuring customer satisfaction using a fuzzy inference system. Journal of Applied Sciences, 9(3), 469-478.

[32] http://rasbt.github.io/mlxtend/user_guide/evaluate/mcnemar/ 